\title{
ÜBERSICHT
}

\section{Stand und Herausforderungen der Aussagepsychologie}

\author{
Max Steller ${ }^{1,2}$
}

Eingegangen: 4. November 2019 / Angenommen: 9. Dezember 2019 / Online publiziert: 30. März 2020

(C) Der/die Autor(en) 2020

\section{Zusammenfassung}

In der jüngeren Vergangenheit ist vermehrt Kritik am Einsatz von Glaubhaftigkeitsbegutachtungen in Verfahren zu Sexualdelikten formuliert worden. Ein Tenor dieser Kritik lautet, dass die wissenschaftlichen Grundlagen der Aussagepsychologie für ihren praktischen Einsatz bei einigen relevanten Fragestellungen für Glaubhaftigkeitsbeurteilungen nicht ausreichend seien. Diese nichtfundierte Kritik stellt keine Herausforderung für die Aussagepsychologie dar, wie der vorliegende Beitrag deutlich macht. Herausforderungen ergeben sich aber aus Gesetzesänderungen in der jüngeren Vergangenheit, wie der Verlängerung von Verjährungsfristen bei sexuellem Kindesmissbrauch oder der Sexualstrafrechtsreform, §177 StGB n.F. Trotz dieser Herausforderungen bringen aussagepsychologische Begutachtungen wissenschaftliche Erkenntnisse in Strafprozesse ein und erfahren in der deutschen Gerichtspraxis eine hohe Akzeptanz. In der Mehrzahl der Fälle leisten Glaubhaftigkeitsbegutachtungen einen Beitrag dazu, dass tatsächlichen Opfern ihr Vorbringen geglaubt wird.

Schlüsselwörter Sexualdelikte $\cdot$ Glaubhaftigkeit $\cdot$ Sachverständigengutachten $\cdot$ Verjährungsfrist $\cdot$ Strafrecht

\section{State and challenges of statement credibility assessment}

\begin{abstract}
In the recent past there has been increased criticism of the inclusion of credibility assessments by expert witnesses in processes with sexual offences. A tenor of this criticism reads that the scientific foundation of statement validity assessment is insufficient for its practical implementation in some relevant cases. This unfounded criticism does not represent a challenge for statement psychology as shown in this article; however, challenges arise from the legislative changes in the recent past, such as the extension of the statutory periods of limitation for criminal prosecutions of child sexual abuse or the reformation of the law governing sexual offences ( $\$ 177$ German Penal Code, StGB, new edition). Despite these challenges, expert opinions about the credibility of witnesses 'statements introduce scientific knowledge into criminal trials and have a high degree of acceptance in German legal practice. In the majority of cases science-based credibility assessments contribute to the fact that assertions of actual victims are believed.
\end{abstract}

Keywords Credibility assessment $\cdot$ Sex offenses $\cdot$ Expert testimony $\cdot$ Limitation period $\cdot$ Crimal law

\section{Einleitung: Glaubhaftigkeitsbegutachtun- gen im Strafprozess}

Prof. Dr. Max Steller

max.steller@aussagepsychologie-berlin.de

1 Zentrum für Aussagepsychologie Berlin, Linienstr. 127, 10115 Berlin, Deutschland

2 Institut für Forensische Psychiatrie, Charité Universitätsmedizin Berlin, Berlin, Deutschland
Das hauptsächliche Anwendungsgebiet der Aussagepsychologie in der deutschen Gerichtspraxis ist die Beurteilung der Glaubhaftigkeit von Anschuldigungen über Sexualdelikte. Bei Aussage-gegen-Aussage-Konstellationen mit Fehlen von Sachbeweisen oder Beobachtungszeugen erhoffen sich Gerichte vom sachverständigen Helfer einen wissenschaftlich begründeten Beitrag zur Wahrheitsfindung. Die Methodik bei inhaltsanalytischen Glaubhaftigkeitsbe- 
gutachtungen ist von verschiedenen Autoren dargestellt worden. ${ }^{1}$

Die Beurteilung des Realitätsgehalts von Zeugenaussagen ist die ureigene Aufgabe von Gerichten, und die Delegation von Verantwortung für richterliche Entscheidungen auf psychologische Sachverständige ist nicht angemessen. Bei einem sachgerechten Einsatz von Sachverständigen erfolgt solch eine Delegation von Verantwortung nicht: Aussagepsychologische Begutachtungen bringen wissenschaftliche Erkenntnisse in Strafprozesse ein. Die ureigene Kompetenz und die Aufgaben von Tatrichtern werden dadurch nicht eingeschränkt, sondern gestärkt. ${ }^{2}$

In einer Bilanz über 4 Jahrzehnte mit Erfahrungen als aussagepsychologischer Sachverständiger wurden verschiedene Entwicklungslinien der Aussagepsychologie dargestellt. ${ }^{3}$ Die Beiträge von herausragenden Vertreterinnen und Vertretern wurden gewürdigt, und die Verwissenschaftlichung und Internationalisierung von ,statement validity analysis" wurden beschrieben. Als Ergebnis wurde eine hohe Akzeptanz von aussagepsychologischer Sachverständigentätigkeit in der deutschen Gerichtspraxis festgestellt.

\section{Glaubhaftigkeitsbegutachtung ist mehr als Inhaltsanalyse}

In der jüngeren Vergangenheit ist vermehrt Kritik am Einsatz von Glaubhaftigkeitsbegutachtungen in Verfahren mit Sexualdelikten formuliert worden. Ein Tenor dieser Kritik lautet, dass die wissenschaftlichen Grundlagen der Aussagepsychologie für ihren praktischen Einsatz bei einigen relevanten Fragestellungen für Glaubhaftigkeitsbeurteilungen nicht ausreichend seien.

Stellvertretend wird auf einen Aufsatz von Schoon und Briken $^{4}$ in einem Themenheft über „Viktimologie, Zeugenschaft und Glaubhaftigkeit" in der Zeitschrift Forensische Psychiatrie, Psychologie und Kriminologie (FPPK) eingegangen. Der ,narrative Beitrag“ (oder auch: die Erzählung über vorliegende Publikationen) liest sich wie die Einar-

\footnotetext{
1 Stellvertretend mit Hinweisen auf andere Publikationen: Volbert R, Steller M (2015) Die Begutachtung der Glaubhaftigkeit. In: Venzlaff/ Förster et al. (Hrsg) Psychiatrische Begutachtung, 6. Aufl, 39. Kap. Urban \& Fischer, München, S 683-707.

2 Daber B (i. V.) § 261 StPO - der Tatrichter und die Aussagepsychologie. In: Deckers R, Köhnken G (Hrsg) Die Erhebung und Bewertung von Zeugenaussagen im Strafprozess. Berliner-Wissenschafts-Verlag, Berlin (erscheint voraussichtlich 2020).

3 Steller M (2013) Vier Jahrzehnte forensische Aussagepsychologie: Eine nicht nur persönliche Geschichte. Prax Rechtspsychol 23:11-32.

${ }^{4}$ Schoon W, Briken P (2019) Zur Anwendbarkeit der Glaubhaftigkeitsbegutachtung unter spezifischen Begutachtungsumständen eine narrative Übersichtsarbeit. Forens Psychiatr Psychol Kriminol 13:125-135.
}

beitung in ein Thema, das den Narratoren zuvor unbekannt war. Er weist diverse Fehler auf.

So wird die merkmalsorientierte Inhaltsanalyse ${ }^{5}$ mit dem Gesamtprozess der Glaubhaftigkeitsbegutachtung gleichgesetzt. Dieser Fehler kam bereits in der internationalen Perzeption von „statement validity assessment“ (SVA) und „criteria-based content analysis“ (CBCA) vor. ${ }^{6}$ Schoon und Briken (a.a.O., S. 126) verwenden diese Begriffe, unterscheiden aber dennoch nicht angemessen zwischen dem diagnostischen (Gesamt-)Prozess „Glaubhaftigkeitsbegutachtung“ (SVA) und dem Teilschritt „Inhaltsanalyse“ (CBCA). Der Aufsatz von Volbert ${ }^{7}$ mit dem Titel „Glaubhaftigkeitsbegutachtung - mehr als merkmalsorientierte Inhaltsanalyse" wurde erwähnt, aber nicht ausreichend berücksichtigt. Es ist immer wieder erstaunlich, ,wie man die aussagepsychologische Methodik verstehen und missverstehen kann"8.

Unter Verweis auf Metaanalysen konstatieren Schoon und Briken, dass Reliabilität und Validität der Merkmalsanalyse ausreichend wissenschaftlich belegt seien (a.a. O., S. 126), für einige Anwendungsbereiche fehle es der Glaubhaftigkeitsbegutachtung aber an Validität. So heißt es u.a., dass ,das derzeitige Begutachtungsverfahren nur eine unzureichende Unterscheidung zwischen suggerierten und erlebnisbasierten Aussagen zulässt“" (a.a.O., S. 133). Diese Behauptung ist falsch.

Zutreffend ist, dass es keine Qualitätsunterschiede zwischen erlebnisbasierten und suggerierten Aussagen gibt. Daher geht es bei ihrer Unterscheidung nicht darum, ob Realkennzeichen vorliegen oder nicht. Das ist nur bei der Prüfung der Lügenhypothese der Fall. Bei der Suggestionshypothese bildet die Analyse der Aussageentwicklung den Schwerpunkt. Inhaltsanalytisch ist zusätzlich zu prüfen, ob gedächtnispsychologisch erwartungswidrige Angaben vorkommen. Suggerierte Falschaussagen sind in der Begutachtungspraxis sehr wohl zu identifizieren. ${ }^{9}$ Nichtfundierte Kritik stellt keine Herausforderung für die Aussagepsycho-

\footnotetext{
5 Steller M, Köhnken G (1989) Criteria-based statement analysis. In: Raskin D (Hrsg) Psychological methods in criminal investigation and evidence. Springer, New York, S 217-245.

6 Steller M (2013) Vier Jahrzehnte forensische Aussagepsychologie: Eine nicht nur persönliche Geschichte. Prax Rechtspsychol 23:16f.

7 Volbert R (2008) Glaubhaftigkeitsbegutachtung - mehr als merkmalsorientierte Inhaltsanalyse. Forens Psychiatr Psychol Kriminol 2:12-19.

8 Volbert R (2009) Glaubhaftigkeitsbegutachtung: Wie man die aussagepsychologische Methodik verstehen und missverstehen kann. Kindesmisshandlung und -vernachlässigung 12(2):52-69.

9 Zum Fehlen von Qualitätsunterschieden zwischen erlebnisbasierten und suggerierten Aussagen bereits Erdmann K (2001) Induktion von Pseudoerinnerungen bei Kindern. Roderer, Regensburg/Überblick zur Suggestionsforschung bei Volbert R (2008) Suggestion. In: Volbert R, Steller M (Hrsg) Handbuch der Rechtspsychologie. Hogrefe, Göttingen, S 331-341/zur aussagepsychologischen Prüfung der Suggestionshypothese in der Praxis: Volbert R, Steller M (2015) s. Fußnote 1.
} 
logie dar, Herausforderungen ergeben sich aber aus Gesetzesänderungen in der jüngeren Vergangenheit.

\section{Herausforderung: Verlängerung von Verjährungsfristen}

Verjährungsfristen beginnen nach Abschluss einer Tat. Im Sexualstrafrecht war - wie bei anderen Delikten - bis 1994 kein Ruhen der Verjährung vorgesehen. Ab 1994 ruhte dann die Verjährung bei sexuellem Kindesmissbrauch erstmals bis zum Eintritt ins 18. Lebensjahr, ab 2009 bis zum Eintritt ins 21. Lebensjahr und seit 2015 bis zum Eintritt ins 30. Lebensjahr. Die Dauer bis zur Verjährung wird durch die Höhe der Strafandrohung bestimmt. Für sexuellen Missbrauch von Kindern gemäß $\S 176$ StGB gilt bei Handlungen ohne Körperberührung eine 5-jährige, bei Handlungen mit Körperberührungen eine 10-jährige und bei schwerem sexuellem Missbrauch ( $\$ 176$ a StGB: mit Eindringen in Körperöffnungen) eine 20-jährige Verjährungsfrist.

Für aussagepsychologische Begutachtungen ergibt sich: Penetrativer sexueller Missbrauch vor dem 14. Geburtstag kann nunmehr von erwachsenen Personen mit einem Alter bis zu 50 Jahren erstmals angezeigt werden. Nach einer Anzeige kann es aus verschiedenen Gründen zum weiteren zeitweiligen Ruhen der Verjährung kommen. Strafprozesse über sexuellen Kindesmissbrauch sind daher auch mit Opfer-Zeugen denkbar, die sich dem Seniorenalter nähern.

Ein sehr langer Zeitablauf zwischen Erlebnis und Erstbekundung erschwert die Glaubhaftigkeitsbegutachtung, macht sie aber nicht unmöglich. Denn ein sexueller Übergriff im Kindesalter, besonders einer mit erfolgter Penetration, ist ein so schwerwiegendes und aus den sonstigen Erfahrungen eines Kindes herausfallendes Erlebnis, dass ein Erinnern daran auch nach Jahrzehnten gedächtnispsychologisch möglich und wahrscheinlich erscheint - von Erlebnissen in der ganz frühen Kindheit einmal abgesehen. Beurteilungen entsprechender Aussagen von Erwachsenen durch aussagepsychologische Begutachtungen sind nicht unmöglich; eine Herausforderung stellen sie allemal dar.

Eine hohe Aussagequalität gilt bekanntlich als Indikator für einen Erlebnishintergrund einer Aussage. Sie ergibt sich allerdings nicht aus dem schematischen Auszählen von Realkennzeichen. Gedächtnispsychologische Kenntnisse müssen immer als Bezugsgröße für die Qualitätseinschätzung einbezogen werden. ${ }^{10}$

Wesentlich ist, dass in der Aussagegeschichte keine Faktoren festzustellen sind, die eine positive Interpretation von hoher Aussagequalität unmöglich machen. Erinnerungssu-

\footnotetext{
${ }^{10}$ Rohmann JA (2018) Erlebnis und Gedächtnis. Charakteristika Mängel - forensische Bedeutung. Eine Übersicht. Prax Rechtspsychol 28:23-59.
}

che mit Erinnerungsarbeit als eigene Aktivität der Aussageperson (z. B. in Selbsthilfegruppen oder in Foren im Internet) oder in Beratungen bzw. Psychotherapien machen den positiven Indikatorgehalt von Qualitätsmerkmalen in Bezug auf einen Erlebnishintergrund von Aussagen zunichte (s. oben). Wenn suggestive Einflüsse feststellbar sind, führt das nicht regelmäßig zu Unentscheidbarkeit. Häufig ergibt sich, dass die Geltungswahrscheinlichkeit für die Suggestionshypothese deutlich höher ist als diejenige für die Erlebnishypothese.

Glaubhaftigkeitsbegutachtungen bei später Erstbekundung über sexuellen Missbrauch sind keine grundsätzlich neuen Herausforderungen. Denn im Sozialrecht, also bei Anträgen nach dem Opferentschädigungsgesetz (OEG), waren solche Fälle seit jeher häufig. Das OEG beinhaltet keine Verjährungsfristen. Hinsichtlich der Beweislast der Antragstellenden gleicht das OEG der Unschuldsvermutung im Strafrecht, wenngleich die Beweisanforderungen in Nuancen anders geregelt sind.

In eigenen Begutachtungen von OEG-Fällen gab es nur einen kleinen Anteil, in denen die Analysen von Aussageentstehung und -entwicklung keine Hinweise auf potenziell suggestive Einflüsse erbrachten. Das ist nicht überraschend, denn in immerhin $50 \%$ von ca. 180 Begutachtungsfällen im OEG-Bereich gaben die Antragstellenden an, dass sie den infrage stehenden sexuellen Missbrauch viele Jahre, ja Jahrzehnte nicht erinnert hatten. Sie hatten ihn im Verlauf von Psychotherapie erstmals erinnert.

Erinnern nach einer langen Zeit des Nicht-Erinnerns ${ }^{11}$ wird in der Literatur als wiedererlangte Erinnerung (,recoverd memory“) nach einer Zeit des Verdrängens (,repressed memory") beschrieben. Wenn Glaubhaftigkeitsbegutachtungen angeordnet werden, steht aber gar nicht fest, dass eine einmal vorhandene, dann aber verlustig gegangene Erinnerung wiedererlangt wurde. Vielmehr geht es um die Fragestellung, ob tatsächlich eine frühere Erinnerung wiedererlangt oder ob eine Scheinerinnerung (,false memory“) generiert wurde. Bei den eigenen OEG-Fällen war die sog. wiedererlangte eine erste Erinnerung, daher wird hier die sperrige Formulierung Erinnern nach Nicht-Erinnern benutzt.

Das Phänomen einer stark verzögerten Erinnerung an Missbrauchserlebnisse ist eng mit dem psychotraumatologischen Konzept über mangelnde Erinnerungsleistungen von Menschen nach traumatischen Erfahrungen verbunden. Das fundamentale Postulat aus der Psychotraumatologie lautet, dass traumatische Ereignisse allenfalls fragmentarisch, also unvollständig, erinnert werden. Ein konsistenter Bericht

\footnotetext{
11 Steller M (2019) Die Entdeckung der Scheinerinnerung. In: Deckers R, Köhnken G (Hrsg) Die Erhebung und Bewertung von Zeugenaussagen im Strafprozess. Berliner-Wissenschafts-Verlag, Berlin, S 71-96.
} 
über ihre Erfahrungen sei Opfern von Traumata daher nicht möglich. ${ }^{12}$ Aus dem Postulat wurden Forderungen nach Modifikation der aussagepsychologischen Realkennzeichen für Aussagen von traumatisierten Personen abgeleitet. ${ }^{13}$ Es wurde eine Umkehr der Realkennzeichen ins Gegenteil gefordert: Je detailärmer, ja je widersprüchlicher oder inhaltlich desaströser eine Aussage sei, desto überzeugender belege sie, dass die Aussageperson das infrage stehende Trauma erlebt habe.

Bereits vor langer Zeit wurde auf die Zirkularität der psychotraumatologischen Argumentation hingewiesen. ${ }^{14}$ Sexueller Missbrauch wird in ihr nicht als infrage stehendes, sondern als feststehendes Ereignis verwendet. Es wird vorausgesetzt, was in Glaubhaftigkeitsbegutachtungen zu prüfen ist. Außerdem wurden die gedächtnispsychologischen Behauptungen bis hin zu vermeintlichen neurobiologischen und -psychologischen Befunden bereits früh durch eine akribische Analyse der entsprechenden Literatur infrage gestellt. ${ }^{15}$

Es gibt also keine sicheren Belege für das Postulat über ein Nicht-Erinnern von Psychotraumata; vielmehr wird beschrieben, dass gerade belastende Erlebnisse besonders gut behalten werden. ${ }^{16}$ Es gibt auch kein durch Außenkriterien gesichertes Wissen darüber, dass ein durch Trauma-

12 Fischer G, Riedesser P (1998) Lehrbuch der Psychotraumatologie, 2. Aufl. Reinhardt, München.

13 Hinckeldey S v, Fischer, G (2002) Psychotraumatologie der Gedächtnisleistung. Reinhardt, München.

14 Steller M (2002) Aussagepsychologie und Strafjustiz: Kooperation ohne Trauma. In: Boetticher A, Huff MW, Landau H, Widmaier G (Hrsg) Sonderheft für Gerhard Schäfer zum 65. Geburtstag am 18. Oktober 2002. Beck, München, S 69-72.

15 Volbert R (2004) Beurteilungen von Aussagen über Traumata. Erinnerungen und ihre psychologische Bewertung. Huber, Bern.

16 Crombag H, Merckelbach H (1997) Mißbrauch vergißt man nicht. Erinnern und Verdrängen - Fehldiagnosen und Fehlurteile. Gesundheit, Berlin.

Auch: Faigman D et al. (2002) Modern scientific evidence. The law and science of expert testimony, vol. 2, ch. 17: repressed memories. West Publishing Co, St. Paul, Minn.

In dieser umfassenden Enzyklopädie von rechtsrelevanten wissenschaftlichen Befunden wurde nach Auswertung einschlägiger Publikationen über die Aussagen von Opfern sexuellen Kindesmissbrauchs festgestellt (a. a. O., S. 357): „None of the victims in any of these studies, as can be judged from the review, was described as having repressed the memory of the abuse experience." In gegenteiligen Darstellungen werde Verdrängen zwar postuliert, aber infantile Amnesie, einfaches Vergessen (beides ohne Wiedererinnern) oder Verschweigen würden die mitgeteilten Daten erklären können. Die Forschungslage ist seit 2002 nicht verändert worden, gegenteilige Behauptungen gibt es allerdings zuhauf.

Dazu auch: Clancy S (2009) The trauma myth. The truth about sexual abuse of children - and its aftermath. Basic Books, New York, Zitat, S. 192: „Generally speaking, the more traumatic an event was when it happened, the more difficult it is for the person to forget about it. Aspects or details of traumatic experiences may be forgotten or distorted (what time of day it was, where the abuse occurred) but not the central part (the abuse).“. therapie erlangtes „Erinnern“, also ein therapiegestütztes Zusammensetzen von Fragmenten, ein Abbild einer längst vergangenen Realität darstellt.

Bereits in den 1990er-Jahren gab es eine umfangreiche wissenschaftliche Literatur über „false memories" ${ }^{17}$. In der Gegenwart waren „Erlebnis und Gedächtnis“"18, der „Einfluss traumatischer Erlebnisse auf das Gedächtnis“"19 bzw. Scheinerinnerungen an Psychotraumata ${ }^{20}$ und ihre Entdeckung durch Glaubhaftigkeitsbegutachtungen ${ }^{21}$ zentrale Themen von aussagepsychologischen Publikationen.

In einer Abhandlung über „Trauma und Justiz“, verfasst von einer Staatsanwältin und einem Psychiater, wird betont: „Erinnerungen an Traumata sind, wie alle Erfahrungen rekonstruktiv: Sie können vollständig wahr sein, wesentliche Ungenauigkeiten enthalten oder, in einigen Fällen, reine Illusionen sein. Kompetente Therapeuten erkennen an, dass Erinnerung fehlbar ist und dass bestimmte therapeutische Ansätze die Wahrscheinlichkeit einer Verzerrung oder Konfabulation erhöhen können“"22. Obwohl über die Vulnerabilität des Gedächtnisses durch Psychotherapie oder andere Einflüsse also wissenschaftlicher Konsens besteht, haben die widerlegten Postulate über die Fragmentierung von Erinnerungen an Psychotraumata und ihre kohärente Zusammensetzung in Traumatherapie hartnäckige Nachhaltigkeit. Sie werden in vielen Publikationen über den Umgang mit dem Verdacht auf sexuellen Kindesmissbrauch wiederholt und stellen daher eine häufige Herausforderung in der Begutachtungspraxis dar. Die Wissenschaft hat längst auf diese Herausforderung reagiert. Sie hat Kenntnisse für die Be-

${ }^{17}$ Loftus E, Ketcham K (1994) The myth of repressed memory: false memories and allegations of sexual abuse. St. Martin's Press, New York, dtsch: Loftus E, Ketcham K (1995) Die therapierte Erinnerung. Vom Mythos der Verdrängung bei Anklagen wegen sexuellen Missbrauchs. Klein, Hamburg.

Ofshe R, Watters E (1994) Making monsters: false memories, psychotherapy, and sexual hysteria: updated with a new final chapter. University of California Press, Berkeley, Los Angeles.

Wakefield H, Underwager R (1994) Return of the furies: an investigation into recovered memory therapy. Open Court, Chicago.

Read J, Lindsay D (1997) Recollections of trauma: scientific evidence and clinical practice. Plenum Press, New York.

$18 \mathrm{Vgl}$. Fußnote 10.

19 Sommer T, Gamer M (2018) Einfluss traumatischer Ereignisse auf das Gedächtnis - neurowissenschaftliche Befunde. Prax Rechtspsychol 28:97-121.

${ }^{20}$ Volbert R (2011) Aussagen über traumatische Erlebnisse. Spezielle Erinnerung? Spezielle Begutachtung? Forens Psychiatr Psychol Kriminol 5:18-31.

Volbert R (2018) Scheinerinnerungen von Erwachsenen an traumatische Erlebnisse und deren Prüfung im Rahmen der Glaubhaftigkeitsbegutachtung: eine rein traumatologische Perspektive ist irreführend. Prax Rechtspsychol 23:54-71.

21 Vgl. Fußnote 11.

${ }^{22}$ Stang K, Sachsse U (2007) Trauma und Justiz. Juristische Grundlagen für Psychotherapeuten - psychotherapeutische Grundlagen für Juristen. Schattauer, Stuttgart, S. 86. 
urteilung von Erinnerungen nach Nicht-Erinnern bereitgestellt.

\section{Noch einmal: Unberechtigte Kritik ist keine Herausforderung}

In dem erwähnten Themenheft der FPPK aus dem Jahr 2019 publizierte ein pensionierter Vorsitzender einer Strafkammer ,aktuelle rechtliche Fragen an die Aussagepsychologie". ${ }^{23}$ Unter Bezug auf die Verlängerung der Verjährungsfrist für schweren sexuellen Missbrauch beklagte er einen Mangel an wissenschaftlichen Erkenntnissen über die Entstehung und die Beurteilung von ,false memories“ (a.a.O., S. 138). ${ }^{24}$ Diese Klage steht allerdings im Gegensatz zur tatsächlichen Fülle entsprechender Literatur (s. oben).

Der juristische Autor will die Überlegenheit einer freien Beweiswürdigung durch Gerichte im Vergleich zu aussagepsychologischen Begutachtungen darlegen. Der Richter a.D. am Landgericht Marburg erwähnt allerdings Folgendes nicht: Der 2. Strafsenat des BGH hat in einem Beschluss vom 29.09.2016 (Az. 2 StR 63/16) die Erwägungen einer Marburger Strafkammer über Scheinerinnerungen als „nicht rechtsfehlerfrei“ beurteilt. Die Kammer hatte in einem Fall mit Erinnern nach Nicht-Erinnern entgegen den Ausführungen einer aussagepsychologischen Sachverständigen verurteilt. Diese Verurteilung hatte vor dem obersten Strafgericht keinen Bestand, da das Landgericht sich nicht ausreichend mit dem Gutachten auseinandergesetzt hatte.

Für die adäquate Einordnung des Beitrags von Wolf ist ein Blick zurück von Interesse. Bereits 1998 berichtete DER SPIEGEL über Fälle mit Erinnerungen von sexuellen Übergriffen nach intensiver Erinnerungsarbeit, die vor ,seiner" Strafkammer verhandelt wurden. ${ }^{25}$ Vergewaltigungsanschuldigungen gegen 3 Männer waren in 200 Sitzungen mit „Wiedererlebenstherapie“ bei einer 23-Jährigen mit Intelligenzminderung evoziert worden. Die Therapeu-

\footnotetext{
${ }^{23}$ Wolf T (2019) Scheinerinnerungen und „false memory“ - aktuelle rechtliche Fragen an die Aussagepsychologie. Forens Psychiatr Psychol Kriminol 13:136-141.

${ }^{24}$ Nach den oben erwähnten Publikationen, aber noch vor dem Aufsatz von Wolf erschienen u. a.:

Stoffels H, Ernst C (2002) Erinnerung und Pseudoerinnerungen. Über die Sehnsucht, Traumaopfer zu sein. Nervenarzt 5:445-450.

Stoffels H (2004) Pseudoerinnerungen oder Pseudologien? Von der Sehnsucht, Traumaopfer zu sein. In: Vollmoeller W (Hrsg) Grenzwertige psychische Störungen. Diagnostik und Therapie in Schwellenbereichen. Thieme, Stuttgart, S 33-45.

Loftus EF (2005) Planting misinformation in the human mind: a 30year investigation of the malleability of memory. Learning Memory 12:361-366.

${ }^{25}$ Friedrichsen G (1998) Ist das jetzt klar, Thomas? DER SPIEGEL Nr. 24, 72-75.
}

tin, die die Erinnerungen nach langer Zeit des Nicht-Erinnerns hervorgeholt hatte, wurde vom Gericht über die Glaubhaftigkeit ihrer Produkte befragt. Als Sachverständiger wurde ein Fachhochschulprofessor a. D. für Mathematik und Physik (sic!) geladen, der sich als Erfinder der Erinnerungstherapie beschrieb. Es kam zu Verurteilungen. Die Gerichtsreporterin bezeichnete es als „,bedeutsam“, dass ein Gericht bereit gewesen sei, ,populistisch aufzugreifen, was der graue Markt der Therapie- und Lebenshilfeszene bietet" (a.a. O., S. 75).

Einleitend wurde ausgeführt: Zur adäquaten Erfüllung der ureigenen Aufgabe von Gerichten und zur Optimierung ihrer Kompetenzen kann es hilfreich sein, geeignete Sachverständige heranzuziehen. Aussagepsychologie ist angewandte Psychologie des Gedächtnisses, daher sind ihre Erkenntnisse für Fälle mit Erinnern nach Nicht-Erinnern relevant. So sah es ja auch der BGH im Gegensatz zur vermeintlich überlegenen Sachkunde einer Marburger Strafkammer.

\section{Kinder- und jugendpsychiatrische Breitbanddiagnostik statt aussagepsychologischer Glaubhaftigkeitsbegutachtung?}

Wenn vermeintliche Kritik an Glaubhaftigkeitsbegutachtungen mit opferzentriertem Pathos vorgetragen wird, ist das besonders befremdlich. Denn dabei wird Folgendes verkannt: Glaubhaftigkeitsbegutachtungen tragen zur Substanziierung der Aussagen von Opfer-Zeugen bei, und es gibt keine anderen wissenschaftlich anerkannten Möglichkeiten zur Überwindung der Unschuldsvermutung bei Fehlen von Beweisen außerhalb der belastenden Aussage. Dennoch titelte ein Professor für Kinder- und Jugendpsychiatrie mit Koautoren im Jahr 2018 in der Zeitschrift Nervenheilkunde wie folgt: „Enormes professionelles Unverständnis gegenüber Traumatisierten. Ist die Glaubhaftigkeitsbegutachtung und ihre undifferenzierte Anwendung in unterschiedlichen Rechtsbereichen eine Zumutung für von sexueller Gewalt Betroffene?" 26 .

Folgendes ist festzustellen (vertiefend ${ }^{27}$ ):

- Das ,professionelle Unverständnis“ von Aussagepsychologen wird aus Berichten über Klagen von Personen ab-

\footnotetext{
${ }^{26}$ Fegert J, Gerke J, Rassenhofer M (2018) Enormes professionelles Unverständnis gegenüber Traumatisierten. Ist die Glaubhaftigkeitsbegutachtung und ihre undifferenzierte Anwendung in unterschiedlichen Rechtsbereichen eine Zumutung für von sexueller Gewalt Betroffene? Nervenheilkunde 7-8:525-534.

27 Volbert R, Schemmel J, Tamm A (2019) Die aussagepsychologische Begutachtung: eine verengte Perspektive? Forens Psychiatr Psychol Kriminol 13:108-124.
} 
geleitet, die eine nichtstützende Glaubhaftigkeitsbegutachtung erlebt hatten. Ob das Ergebnis der Begutachtungen zutreffend war oder nicht, bleibt im Beitrag ungeprüft.

- Der Beitrag enthält extrem widersprüchliche Darstellungen. Neben Diskreditierung der Aussagepsychologie beschreibt der Erstautor, dass er in „wichtigen“ Fällen bei eigenen Begutachtungen von der Aussagepsychologie Gebrauch gemacht habe. Ein Beispielsfall mit Wichtigkeit betrifft einen angeklagten Arzt. Sind Anklagen gegen andere Personen weniger wichtig?

- Die Unterstellung einer undifferenzierten Anwendung von Aussagepsychologie in unterschiedlichen Rechtsbereichen wird weder dargestellt noch begründet, vielmehr werden die (widerlegten) psychotraumatologischen Behauptungen wiederholt.

- Eine Alternative zur aussagepsychologischen Begutachtung bei infrage stehender Traumatisierung durch sexuelle Übergriffe wird nicht vorgestellt.

Für die Autoren scheint die Unschuldsvermutung keine zivilisatorische Errungenschaft, sondern im Sexualstrafrecht eine „Zumutung“ für Opfer-Zeugen zu sein. OpferZeuge ist ein Kürzel für Belastungszeugen, deren Anschuldigung ein Opfer-Erlebnis enthält. Verurteilungen können aber erst nach Feststellung der Glaubhaftigkeit ihrer Anschuldigung durch ein Gericht erfolgen - ggf. unter Mitarbeit eines aussagepsychologischen Sachverständigen.

Das Unverständnis der Autoren für Beweislast sowie für Methodik und Funktion von aussagepsychologischen Begutachtungen wäre nicht der Kommentierung wert, wenn sich nicht Identisches in einer Publikation der „Unabhängigen Kommission zur Aufarbeitung sexuellen Kindesmissbrauchs" finden würde. In einer Broschüre aus dem September $2018^{28}$ wird verlautbart, dass aussagepsychologische Sachverständige und „Entscheider in der Justiz“ unsensibel für Traumafolgen seien. Der Unschuldsvermutung wird eine andere Maxime gegenübergestellt; nämlich der Grundsatz „im Zweifel für das Opfer“ (a.a.O., S. 23). Wie Opfer-Sein ohne Glaubhaftigkeitsprüfung juristisch festgestellt werden soll, wird nicht gesagt.

Es ist zu befürchten, dass unberechtigte Diskreditierungen der Aussagepsychologie dazu führen können, intuitiven Glaubhaftigkeitsattributionen Vorschub zu leisten. Eine Folge kann auch darin bestehen, dass tatsächlichen Opfern die Unterstützung durch Glaubhaftigkeitsbegutachtungen ver-

\footnotetext{
${ }^{28}$ Unabhängige Kommission zur Aufarbeitung sexuellen Kindesmissbrauchs (2018) Empfehlungen zur Verbesserung der Situation von sexueller Gewalt in Kindheit und Jugend betroffener Menschen in Ermittlungs- und Strafverfahren. www.aufarbeitungskommission.de.

29 Niehaus S (2018) Im Interesse kindlicher Opfer. Prax Rechtspsychol 28(2):99-120.
}

sagt wird. Niehaus ${ }^{29}$ wies bereits darauf hin, dass die von Fegert et al. vorgetragene Kritik an Glaubhaftigkeitsbegutachtungen nicht „im Interesse kindlicher Opfer“ sei.

Auch hier ist ein Blick zurück erhellend: Bereits vor 20 Jahren reklamierten kinder- und jugendpsychiatrische Verbände die Begutachtung kindlicher Opfer-Zeugen für ihre Profession (Stellungnahme zur Glaubwürdigkeitsbegutachtung 1999)..$^{30}$ Gefordert wurden eine Breitbanddiagnostik unter Einsatz medizinischer Apparate, u. a. die routinemäBige Durchführung von elektroenzephalographischen Untersuchungen und die Anwendung psychiatrischer Diagnoseschemata auf kindliche Opfer-Zeugen; auch auf solche, bei denen psychische Folgeschäden des infrage stehenden sexuellen Missbrauchs noch gar nicht erkennbar waren. Die Ausführungen der kinder- und jugendpsychiatrischen Verbände blieben nicht ohne Kritik ${ }^{31}$, sie blieben aber ohne durchschlagende Wirkung: Pathologisierungen kindlicher Opferzeugen und ihre routinemäßigen Traumatisierungen durch breitbandige Apparatemedizin fanden nicht statt.

Die Federführung für die Stellungnahme der kinder- und jugendpsychiatrischen Verbände im Jahr 1999 hatte der Erstautor des Beitrags über das ,professionelle Unverständnis“ aufseiten von Aussagepsychologen. Das berufsständische Interesse bei der Betonung kinder- und jugendpsychiatrischer Aspekte für Glaubhaftigkeitsbegutachtungen wird überdeutlich; eine ernst zu nehmende Herausforderung für die Aussagepsychologie stellt die Kritik von Fegert et al. aus dem Jahr 2019 nicht dar.

\section{Herausforderung: Sexualstrafrechtsreform, § 177 StGB n. F.}

Am 10.11.2016 trat das „Fünfzigste Gesetz zur Änderung des Strafgesetzbuches - Verbesserung des Schutzes der sexuellen Selbstbestimmung“ in Kraft. Der 13. Abschnitt des StGB wurde mit Blick auf die „Nein-heißt-Nein“-Debatte grundlegend reformiert. Die Realisierung dieser Reform wurde durch die Ereignisse in der Kölner Silvesternacht 2015/2016 beschleunigt. Auch das eigenartige Strafverfahren, das durch eine Prominente namens Gina-Lisa Lohfink angestoßen worden war, hatte wahrscheinlich eine katalysierende Wirkung. ${ }^{32}$ Die Reform führte zu mehreren Geset-

\footnotetext{
30 Stellungnahme zur Glaubwürdigkeitsbegutachtung der Deutschen Gesellschaft für Kinder und Jugendpsychiatrie (DGKJP) und der Bundesarbeitsgemeinschaft der Leitenden Klinikärzte für Kinder- und Jugendpsychiatrie und Psychotherapie (1999). Z Kinder- und Jugendpsychiatr Psychother 27(1):72-75.

31 Steller M, Volbert R (2000) Anforderungen an die Qualität forensisch-psychologischer Glaubhaftigkeitsbegutachtungen Das BGH-Urteil vom 30. Juli 1999. Prax Rechtspsychol 10(1):102-116.

32 Niehaus S, Reutemann M (2017) Editorial zum Themenheft „Sexualstrafrechtsreform“. Prax Rechtspsychol 27:5-6.
} 
zesänderungen; hier wird lediglich auf Teile des neu gefassten $\S 177$ StGB eingegangen. Diese Vorschrift betrifft sexuelle Übergriffe, sexuelle Nötigung und Vergewaltigung.

Nach altem und neuem Gesetz war und ist die Durchführung einer sexuellen Handlung ohne Einwilligung der anderen Person die Voraussetzung für die Strafbarkeit. Die Reform betont aber einen wichtigen Paradigmenwechsel: Statt Nötigung oder Widerstand steht nunmehr der ,entgegenstehende Wille“ im Zentrum der Betrachtung. Kritikwürdig an der alten Fassung erschien nämlich, dass sie bestimmte Konstellationen aus der Lebenswirklichkeit nicht explizit erfasste. Es wird u. a. argumentiert: Wenn ein Opfer aus verschiedenen Gründen auf körperlichen Widerstand verzichtet, und wenn eine offenkundige Nötigung nicht vorkommt, also keine Nötigung mit der Durchführung oder expliziten Androhung von Gewalt stattfindet, seien solche Fälle durch das Raster der Strafbarkeit gefallen. Zum Teil wird allerdings entgegengehalten, dass eine solche "Schutzlücke“ in der alten Fassung vom $\S 177$ StGB gar nicht bestanden habe; auch die frühere Rechtsprechung habe das subjektive Empfinden von Opfern berücksichtigt. Es sei auch früher bereits verurteilt worden, wenn der ,entgegenstehende Wille" (gegen die Durchführung sexueller Handlungen) in irgendeiner Weise äußerlich erkennbar gemacht worden sei. ${ }^{33}$

Der entgegenstehende Wille kann durch verbale Äußerungen, aber auch „konkludent“, also durch Weinen, Abwehren u. a. zum Ausdruck gebracht worden sein. Aus aussagepsychologischer Sicht ergibt sich dazu: Bei der Beurteilung einer Aussage über eine infrage stehende Vergewaltigung war der Umfang der zu analysierenden Explorationsinhalte schon immer relativ gering. Im Zentrum der aussagepsychologischen Exploration steht/stehen nicht - wie beim sexuellen Kindesmissbrauch - die Schilderung der sexuellen Handlung an sich, sondern die Schilderungen der umstrittenen Aussageteile, also derjenigen über Widerstand oder Nötigung (bereits Michaelis-Arntzen ${ }^{34}$ ). Ein relativ geringer Aussageumfang stellte schon immer eine Erschwerung für aussagepsychologische Begutachtungen dar. Diese Herausforderung könnte in solchen Fällen noch gröBer werden, die nach der neuen Fassung des § 177 StGB zu behandeln sind. Im Hinblick auf $\S 177$ Abs. 1 n. F., in dem es um den ,erkennbaren Willen“ eines potenziellen Opfers geht, könnte allerdings auch alles beim Alten bleiben. Denn für die Erkennbarkeit spielen ja - wie bisher - Schilderungen über Abwehrhandlungen, Drohungen oder Gewaltanwendungen eine Rolle; ,konkludente“ Ablehnun-

\footnotetext{
33 Röttgering D, Lechtenberg F (2018) Die Reform des Sexualstrafrechts nach dem 50. StrÄndG. In: Deckers R, Köhnken G (Hrsg) Die Erhebung und Bewertung von Zeugenaussagen im Strafprozess, 3. Bd. Berliner-Wissenschafts-Verlag, Berlin, S 207.

${ }^{34}$ Michaelis-Arntzen E (1994) Die Vergewaltigung aus kriminologischer, viktimologischer und aussagepsychologischer Sicht. 2. Aufl. Beck, München, S 43.
}

gen sind nunmehr durch den Paradigmenwechsel eindeutig im Gesetz verankert.

Eine deutliche Herausforderung an die Aussagepsychologie ist allerdings im Abs. 2. der neuen Fassung von $§ 177$ StGB enthalten. In ihm sind 5 Tatbestandvarianten definiert. Es geht jeweils um die Obliegenheit des Opfers, den entgegenstehenden Willen zu kommunizieren bzw. um Einschränkungen der Fähigkeit zur entsprechenden Kommunikation.

So wird bestraft, wenn ein Täter folgende Konstellationen ausnutzt: Im Abs. 2, Nr. 1 die Unmöglichkeit für Willensbildungen oder-äußerungen durch Schlaf, Vollnarkose, K.-o.-Tropfen u.a. und in Nr. 2 starke Einschränkungen wie geistige Behinderung, Demenz oder starke Trunkenheit. In diesen Fällen kann retrospektive Psychodiagnostik bei Opfer-Zeugen von Wert sein. Aussagepsychologische Inhaltsanalysen werden dagegen keine Rolle spielen, da in den genannten Zuständen oder mit den genannten Eigenschaften gar keine analysierbaren Aussagen von Personen erhalten werden können.

Aussagepsychologische Beurteilungen von „Überrumpelungen“ (Abs. 2, Nr. 3) per Inhaltsanalysen dürfte wegen des sehr eingeschränkten Umfangs des Aussagematerials erhebliche Schwierigkeiten bereiten.

Die Nötigung per Bedrohung enthält Abs 2, Nr. 5; dies gleicht hinsichtlich der Begutachtung den Anforderungen aus dem Abs. 1. Eine wirkliche Herausforderung besteht in Beurteilungen darüber, ob eine Person jegliche Abwehrhandlungen oder -äußerungen unterlassen hat, weil ihr aus subjektiver Sicht ein „empfindliches Übel“ gedroht hätte, wenn sie Entsprechendes getan hätte. Diese Konstellation ist in Abs. 2, Nr. 4 enthalten. Gewalterfahrungen in der Vergangenheit können natürlich das Handeln bzw. Unterlassen einer Person in der zu beurteilenden Situation bestimmt haben. Es geht also um Fallkonstellationen, bei denen ein Zeitverzug zwischen erfahrener Gewalt und perzipiertem zukünftigen Übel besteht, und in denen der Vorgang gewaltfrei, aber nicht einvernehmlich verläuft. Aber können Schilderungen über ein Gewährenlassen aus Furcht vor einem Übel überhaupt inhaltsanalytisch beurteilt werden?

Die aussagepsychologische Analyse stellt auf die Herausarbeitung von inhaltlichen Merkmalen ab, deren Erfindung einer Aussageperson mangels ausreichender Kompetenz nicht zugetraut wird. Die Interpretation dieses $\mathrm{Ab}$ gleichs von Aussagequalität und (Erfindungs-)Kompetenz wird erschwert, wenn bei der Aussageperson Parallelerlebnisse gegeben waren: Eine Übertragung von Erfahrungen auf eine andere Situation ist eine einfachere (Lügen-)Leistung als die vollkommene Erfindung.

In der Konstellation, die in $\S 177$ StGB n. F. Abs. 2, Nr. 4 beschrieben wird, ist die Ausgangslage für Inhaltsanalysen noch schwieriger: Selbst wenn die Schilderungen über frühere Gewalterfahrungen detailreich und anschaulich er- 
folgen, also auf einen Erlebnishintergrund verweisen, lässt dies noch keinen Schluss zu, dass sie sich auf Äußerungsfähigkeit und Verhalten in einer neuen Situation ausgewirkt haben. Um die Beurteilungsgrundlage zu verbreitern, kann es nötig werden, eine Erforschung der Persönlichkeit der Aussageperson und ihrer gesamten Vita (einschließlich der Vita sexualis) vorzunehmen, die weit über das hinausgeht, was auch bisher als Bezugsgröße für die Inhaltsanalyse nötig war.

Wenn umfassende Ausforschungen der Aussageperson durch biografische und tatbezogene Explorationen in Ermittlungs- und Strafverfahren nötig werden, kann das dazu führen, ,dass vermehrt aussagepsychologische Gutachten eingeholt werden, um eine breitere Beweisgrundlage zu erhalten“, prognostiziert ein Strafverteidiger. ${ }^{35}$ Er befürchtet allerdings, dass die Aussagepsychologie an der Beantwortung der Fragestellung aus $\S 177$ StGB n.F. Abs. 2, Nr. 4 „scheitern“ wird (a.a.O., S. 285 sowie bereits Lederer und Deckers $\left.^{36}\right)$.

Die Beurteilung von Schilderungen über einen entgegenstehenden Willen, der aufgrund vergangener Gewalterfahrungen der Aussageperson weder explizit noch konkludent geäußert wurde, stellt für die Justiz und für die Aussagepsychologie auf jeden Fall eine enorme Herausforderung dar. Rohmann ${ }^{37}$ betonte dazu, dass die Aussagepsychologie ihre Grenzen erkennen und bewahren müsse.

\section{Herausforderungen in der Realität}

Es stellt sich die Frage, ob im Strafrecht eine Zunahme von Fällen festzustellen ist, in denen es zu Anzeigen mit sehr langer Verzögerung nach der angegebenen Tatzeit gekommen ist. Die Polizeiliche Kriminalstatistik (PKS) des Bundeskriminalamtes verzeichnete 12.321 Fälle des sexuellen Kindesmissbrauchs für das Jahr 2018. Dies entspricht einem Anstieg von 6,7\% gegenüber 2017 mit 11.547 Anzeigen. Dass dieser Anstieg auf vermehrte Anzeigen von Erwachsenen über lange zurückliegende Taten begründet ist, erscheint sehr fraglich. Denn die Zahl aus dem Jahr 2018 liegt nur geringfügig über derjenigen im Jahr 2016 mit 12.019 Anzeigen. Die genannten Schwankungen entsprechen außerdem dem jahrelangen Verlauf. Aus den Häu-

\footnotetext{
35 Deckers R (2018) Der aussagepsychologische Realkennzeichenkatalog von Steller/Köhnken (1989) aus juristischer Sicht. In: Barton S, Fischer T, Jahn M, Park T (2018) Festschrift für Reinhold Schlothauer zum 70. Geburtstag. Beck, München, S 284.

36 Lederer J, Deckers R (2017) Verteidigung mit Blick auf die Integration (aussage-)psychologischen Sachverstands in das Strafverfahren nach der Gesetzesnovelle einiger Normen des Sexualstrafrechts (§§ 177, 184 i und j StGB). Prax Rechtspsychol 27(1):75-78.

37 Rohmann JA (2017) Das neue Sexualstrafrecht - eine aussagepsychologische Perspektive. Prax Rechtspsychol 27(1):43.
}

figkeitszahlen nach 2015, dem Jahr mit der bisher letzten Verlängerung von Verjährungsfristen, ist daher bisher keine relevante Zunahme von Fällen mit Anzeigen von Erwachsenen über weit zurückliegenden sexuellen Kindesmissbrauch abzuleiten. Sollte ein solcher Anstieg in Zukunft eintreten, ist die Aussagepsychologie aufgrund der Forschungslage und der praktischen Erfahrungen im Sozialrecht gut aufgestellt.

Für evtl. Veränderungen der Häufigkeit von Fällen, die unklare Kommunikationen zum Gegenstand haben, sind gemäß dem Wissen des Autors keine statistischen Zahlen vorhanden. Für ,,Vergewaltigung, sexuelle Nötigung, sexuelle Übergriffe“, also für $\S 177$ StGB insgesamt, wurden 2017 , dem Jahr nach der Gesetzesreform, in der PKS 11.282 Anzeigen aufgeführt. Das wirkt wie ein enormer Anstieg gegenüber 2016 mit 7919 Anzeigen für sexuelle Nötigung und Vergewaltigung. Allerdings ist in der $P K S$ ausdrücklich vermerkt, dass ein Vergleich dieser Zahlen wegen der Gesetzesänderungen nicht möglich ist.

Für das Jahr 2018 wurden 9324 Anzeigen gemäß $§ 177$ StGB registriert. Das würde einen Rückgang von 18,2\% gegenüber der hohen Zahl des Jahres 2017 und eine deutliche Annäherung an die Anzeigehäufigkeit vor der Strafrechtsreform signalisieren. In der Einleitung zur PKS 2018 steht noch einmal, dass ein direkter Vergleich der Zahlen von 2016 bis 2018 nicht möglich ist, da verschiedene frühere Tatbestände in den Statistiken erst nach und nach zusammengeführt wurden.

Als Fazit ergibt sich: Ein Anstieg von Fallkonstellationen, die mit Aufnahme der „Nein-heißt-Nein“-Begründung in $\S 177 \mathrm{StGB}$ n.F. im Zusammenhang stehen können, ist derzeit nicht zu erkennen. Gleichwohl gilt, dass aussagepsychologische Wissenschaft und Praxis sich auf die neuartigen Fallkonstellationen einstellen müssen.

\section{Doppelter Opferschutz durch Glaubhaftigkeitsgutachten}

Nach Justizkatastrophen in den 1990er-Jahren (Stichwörter: Wormser Prozesse vor dem Landgericht Mainz und Montessori-Prozess vor dem Landgericht Münster) erteilte der 1. Strafsenat des BGH eklektisch-intuitiven Glaubhaftigkeitsbeurteilungen eine Absage. Nur der aussagepsychologischen Methodik wurde Beweiswert zuerkannt (BGH St 45, 164). Seit dem BGH-Urteil vom 30.07.1999 über „Wissenschaftliche Anforderungen an aussagepsychologische Begutachtungen (Glaubhaftigkeitsgutachten)" sind 2 Jahrzehnte vergangen. Es waren 2 Jahrzehnte der erfolgrei-

\footnotetext{
38 Vgl. Fußnote 34.
} 
chen Zusammenarbeit von Gerichten mit aussagepsychologischen Gehilfen (z. B. Deckers ${ }^{38}$ und bereits Deckers ${ }^{39}$ ).

In einem Beschluss des 3. Strafsenats des BGH vom 03.05.2019 (Az. 3 StR 462/18) wurde der Stellenwert von Glaubhaftigkeitsgutachten noch einmal ausdrücklich hervorgehoben. Ein Landgericht hatte aus einem Glaubhaftigkeitsgutachten lediglich die Ausführungen zur Aussagetüchtigkeit, aber nicht zur Glaubhaftigkeit der Aussage einer Belastungszeugin berücksichtigt. Der BGH-Senat führte dazu aus: „Die Aussageanalyse, ..., ist aber Bestandteil eines wissenschaftlich fundierten Glaubhaftigkeitsgutachtens ..., den das Gericht aus Gründen der Aufklärungspflicht zur Kenntnis zu nehmen und dessen Inhalt es im Urteil darzulegen hat (BGH, Beschluss vom 04.09.2002 - 2 StR 307/02, BGHR StPO $§ 261$ Beweiskraft 4)“. Die einleitend beschriebene Akzeptanz von aussagepsychologischen Gutachten wurde in diesem Beschluss des BGH also erneut bestätigt.

Angesichts unberechtigter Kritik ist (erneut) darauf hinzuweisen, dass Glaubhaftigkeitsbegutachtungen in der Mehrzahl der Fälle einen Beitrag dazu leisten, dass tatsächlichen Opfern ihr Vorbringen geglaubt wird. Für die Überwindung der Hürde „Unschuldsvermutung“ vor einer Verurteilung wurde vor 20 Jahren vor dem BGH die Metapher benutzt, es gehe um die Zurückweisung der Nullhypothese ${ }^{40}$. Diese an die Prüfstatistik angelehnte Formulierung mag ,didaktisch unangemessen“ sein $^{41}$, da sie zuweilen oder häufig missverstanden wird. Andererseits wurde die Aufmerksamkeit auf die wissenschaftlich und juristisch nötige Falsifikationsstrategie gelenkt.

Auf die Metapher „Nullhypothese“ kann verzichtet werden. Angesichts der berichteten pseudokritischen Beiträge über Glaubhaftigkeitsbegutachtungen erscheint es aber keineswegs überflüssig, Unschuldsvermutung und Beweislast immer wieder $\mathrm{zu}$ betonen. Professionelles Unverständnis für Notwendigkeit und Effizienz von Glaubhaftigkeitsbegutachtungen ist eine Zumutung. Es verstärkt die Befürchtung, dass im Sexualstrafrecht die Gefahr besteht, es könne unschuldig verurteilt werden. ${ }^{42}$

Glaubhaftigkeitsbegutachtungen stellen in jedem Einzelfall intellektuelle Herausforderungen dar. Ihre ethische Vertretbarkeit ist dadurch begründet, dass a priori keineswegs feststeht, ob ein Gutachtenergebnis dazu beitragen wird, ein tatsächliches Opfer zu stützen oder ein Fehlurteil zu vermeiden.

Funding Open Access funding provided by Projekt DEAL.

Interessenkonflikt M. Steller gibt an, dass kein Interessenkonflikt besteht.

Open Access Dieser Artikel wird unter der Creative Commons Namensnennung 4.0 International Lizenz veröffentlicht, welche die Nutzung, Vervielfältigung, Bearbeitung, Verbreitung und Wiedergabe in jeglichem Medium und Format erlaubt, sofern Sie den/die ursprünglichen Autor(en) und die Quelle ordnungsgemäß nennen, einen Link zur Creative Commons Lizenz beifügen und angeben, ob Änderungen vorgenommen wurden.

Die in diesem Artikel enthaltenen Bilder und sonstiges Drittmaterial unterliegen ebenfalls der genannten Creative Commons Lizenz, sofern sich aus der Abbildungslegende nichts anderes ergibt. Sofern das betreffende Material nicht unter der genannten Creative Commons Lizenz steht und die betreffende Handlung nicht nach gesetzlichen Vorschriften erlaubt ist, ist für die oben aufgeführten Weiterverwendungen des Materials die Einwilligung des jeweiligen Rechteinhabers einzuholen.

Weitere Details zur Lizenz entnehmen Sie bitte der Lizenzinformation auf http://creativecommons.org/licenses/by/4.0/deed.de.

Prof. Dr. Max Steller Universitätsprofessor für Forensische Psychologie a. D., Fachpsychologe für Rechtspsychologie BdP/DGPs

\footnotetext{
42 Steller M (2015) Nichts als die Wahrheit? Warum jeder unschuldig
} verurteilt werden kann. Heyne, München.
39 Deckers R (2007) Vorwort. In: Deckers R, Köhnken G (Hrsg) Die Erhebung und Bewertung von Zeugenaussagen im Strafprozess. Berliner-Wissenschafts-Verlag, Berlin, S VII-XIII.

40 Steller M, Volbert R (1999) Wissenschaftliches Gutachten, forensisch-aussagepsychologische Begutachtung. Prax Rechtspsychol 9(2):61.

${ }^{41}$ Greuel L (2009) Was ist Glaubhaftigkeitsbegutachtung (nicht)? Zum Problem der Dogmatisierung in einem wissenschaftlichen Diskurs. Kindesmisshandlung und -vernachlässigung 12(2):80. 